\title{
Étude expérimentale de l'influence de la force de Coriolis sur la propagation de la marée dans la Manche
}

\section{Experimental study of the influence of the Coriolis force on the tide propagation in the English Channel}

\author{
PAR R. BONNEFILLE
}

INGÉNIEUR AU LaBORatome NatronaL D'HYDraUliove

\begin{abstract}
Le petit canal tournant construt au Laboratoire National d'Hydranlique de Chaton a pour but d'étudier l'influence relative de la force de Coriolis sur les marées de la Manche, dans le cadre des études préliminaires de l'aménagement de l'usine marémotrice des illes Chausey. Le canal représente schématiquement la Manche par deux bassins rectangulaires contigus. Sa longueur est de $120 \mathrm{~cm}$, sa largeur de $30 \mathrm{~cm}$. La profondeur de l'eau est constante et égale à $5 \mathrm{~cm}$, la période de la marée est de 3 secondes, la vitesse de rotation de la plate-forme peut atteindre 1 tour en 4 secondes.

L'étude de la propagation des ondes-marée dans le canal à fond lisse, et avec différentes conditions aux limites, a permis d'observer la transformation d'une ligne nodale en un point amphidromique lors de la rotation du canal. L'étude des trajectoires de petits flotteurs a mis en evidence une circulation des courants s'effectuant dans le même sens que la rotation alu bassin.

Les mêmes études effectuées dans le canal comportant un fond rugueux, ont montré l'influence simultané de la force de Coriolis et des forces de frottement sur le phénomène de la marée en Manche. On a pu obtenir une disparité entre les marées des cóles anglaises et frangaises, ainsi qu'un point amphiltromique intuel.
\end{abstract}

The small rotating flume, built at the Laboratoire National d'Hydraulique at Chatou, is designed to investigate the relative influence of the Coriolis force on tides in the English Channel in connection with preliminary studies for the Chausey Islands tidal power station scheme.

The flume represents the Channel schematically by means of two adjoining rectangular tanks. Its length is $120 \mathrm{cms}$ and its width $30 \mathrm{cms}$. The water depth is constant at $5 \mathrm{cms}$, the tide period is 3 seconds and the rotational speed of the platform can be one turn in four seconds.

Investigation of tide wave propagation in the smooth floored flume, using various boundary conditions, has ailowed the transformation of nodal line into an amphidromic point to be observed when the flume is rotated. Observation of the paths taken by small floats has brought to light a circulation of the currents in the same direction as that in which the tank is rotating.

The same tests carried out in the flume having a rough floor has shown the simultaneous in." fluence of the Coriolis and frictional forces on the phenomenon of the Channel tide. It has been possible to obtain a disparity between the tides on the English and French coasts, as well as a virtual amphidromic point.

\section{I. - INTRODUCTION}

Le problème des marées envisagé du point de vuc hydraulique est l'un des plus intéressants par la grandeur de l'échelle des mouvements mis en jeu et de par la complexité que met on lumière l'analyse du phénomène. Bien que le problème ait été depuis longtemps mis en équa- tion, aucune solution numérique ne peut être facilement obtenue et ceci même dans les cas simples par suite de l'introduction du terme de Coriolis. Notre connaissance des marées océaniques et de celles des mers littorales a essentiellement sa source dans l'observation. Or cette 
étude est difficile dans le cas des marées océaniques, et pratiquement seules'les marées des mers littorales sont bien connues.

Du point de vue de l'ingénieur, la connaissance des marécs se pose dans le cas où des travaux à la mer sont susceptibles de modifier le régime des marées. La question vient à l'ordre du jour avec les projets de construction des centrales marémotrices de grande puissance.

Le problème étant difficilement soluble par le calcul seul, la solution peut être demandée au modèle réduit. Or, la représentation de la force de Coriolis à l'échelle suppose le modèle réduit animé d'une vitesse de rotation d'autant plus rapide que les échelles de similitude sont petites. On se trouve ainsi conduit à résoudre un problème d'ordre technologique très complexe : assurer la rotation d'une plate-forme de grand diamètre en évitant toutes les vibrations parasites susceptibles de troubler le phénomène hydraulique de la marée sur le modèle réduit. Cette difficulté amène à chercher d'autres procédés de représentation de la force de Coriolis sur modèle réduit ou bien à s'affranchir de cette représentation en admettant une certaine approximation dans la reproduction du phénomène. Cette dernière éventualité sera d'autant plus valable que les autres forces entrant en jeu sont importantes : dans les mers littorales, l'influence du frottement peut être primordiale.

Dans le cas particulier de l'aménagement marémoteur des illes Chausey, une partie des études préliminaires consiste à déterminer la perturba- tion apportée à la marée en Manche par la présence et le fonctionnement de l'usine. A cet effet, on a voulu d'abord s'affranchir de la reproduction de la force de Coriolis. Le Laboratoire National d'Hydraulique a construit un modèle réduit non tournant de la Manche [1], au 1/50 000 en plan et 1/500 en hauteux. La période de la marée semi-diurne sur ce modèle est de 20 secondes. Pour que la force de Coriolis soit représentée en similitude, le modèle devrait tourner à raison de 1 tour en 51 secondes.

Or, le modèle étant fixe, il n'a pas été possible d'obtenir une représentation correcte de la marée sur la totalité du domaine maritime. La force de Coriolis est donc un parametre important dans le phénomène de la marée en Manche. Il convient cependant de préciser la validité de cette hypothèse et, par voie de conséquence, la validité des résultats acquis sur le modèle réduit non tournant. Il a alor's été envisagé d'étudier l'influence de la force de Coriolis sur la marée en utilisant une petite cuve susceptible d'être animée d'un mouvement de rotation. La comparaison des ondes-marées obtenues avec ou sans force de Coriolis, et avec ou sans frottement, doit permettre de préciser les influences relatives de la force de Coriolis et du frottement et d'en tirer des conséquences sur la possibilité de construire des modìles à marée non tournants. Nous exposerons ici quelques-uns des premiers résultats de celte élude en nous limitant au cas de l'influence de la foree de Coriolis dans la Manche.

\section{II. - DESCRIPTION DE L'INSTALLATION}

Le canal d'essais est posé sur une plate-forme tournante (voir photographie) de $4 \mathrm{~m}$ de diamitre, et pouvant supporter une charge de $1500 \mathrm{~kg}$ excentrée de $30 \mathrm{~cm}$. La vitesse de rotation peut varier de facon continue entre 1 tour en $10 \mathrm{~s}$ et 1 tour en $2 \mathrm{~s}$ (fig. 1 ).

Le canal est constitué par une cuve rectangulaire à parois en plexiglas de hauteur $10 \mathrm{~cm}$. A l'intérieur du bassin, peuvent être disposés des éléments de parois schématisant diverses configurations géométriques des côtes. L'axe de rotation de la plate-forme passe à proximité du centre du canal, de facon à rendre minimum la courbure de la surface libre utile, sous l'action de la force centrifuge.

Le générateur de marée est constitué par une pelle de batteur, animée soit d'un mouvement dé translation de course réglable de 0 à $20 \mathrm{~cm}$, et variant sinusoïdalement en fonction du temps, soit d'un mouvement de pivotement autour d'un axe vertical quelconque.

Les appareils de mesure utilisés sont:
- Pour les mesures de marnage : un limnigraphe a pointe vibrante asservie dont le palpeur se déplace d'un mouvement lent (1 m en $4 \mathrm{mn}$ ) le long d'un rail parallèle au grand côté du canal. On enregistre ainsi directe-

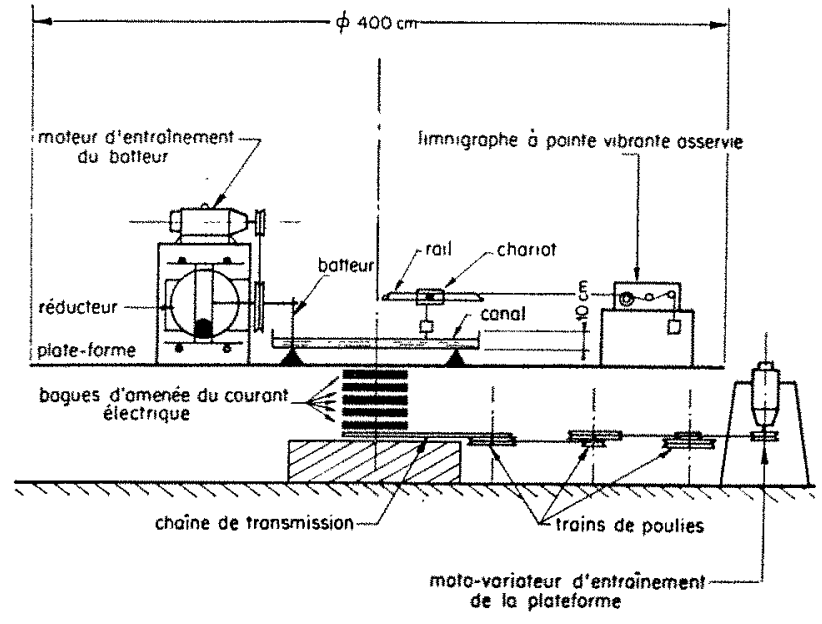

FIa. 1. - Schéma de linstallation du canal tournant. 


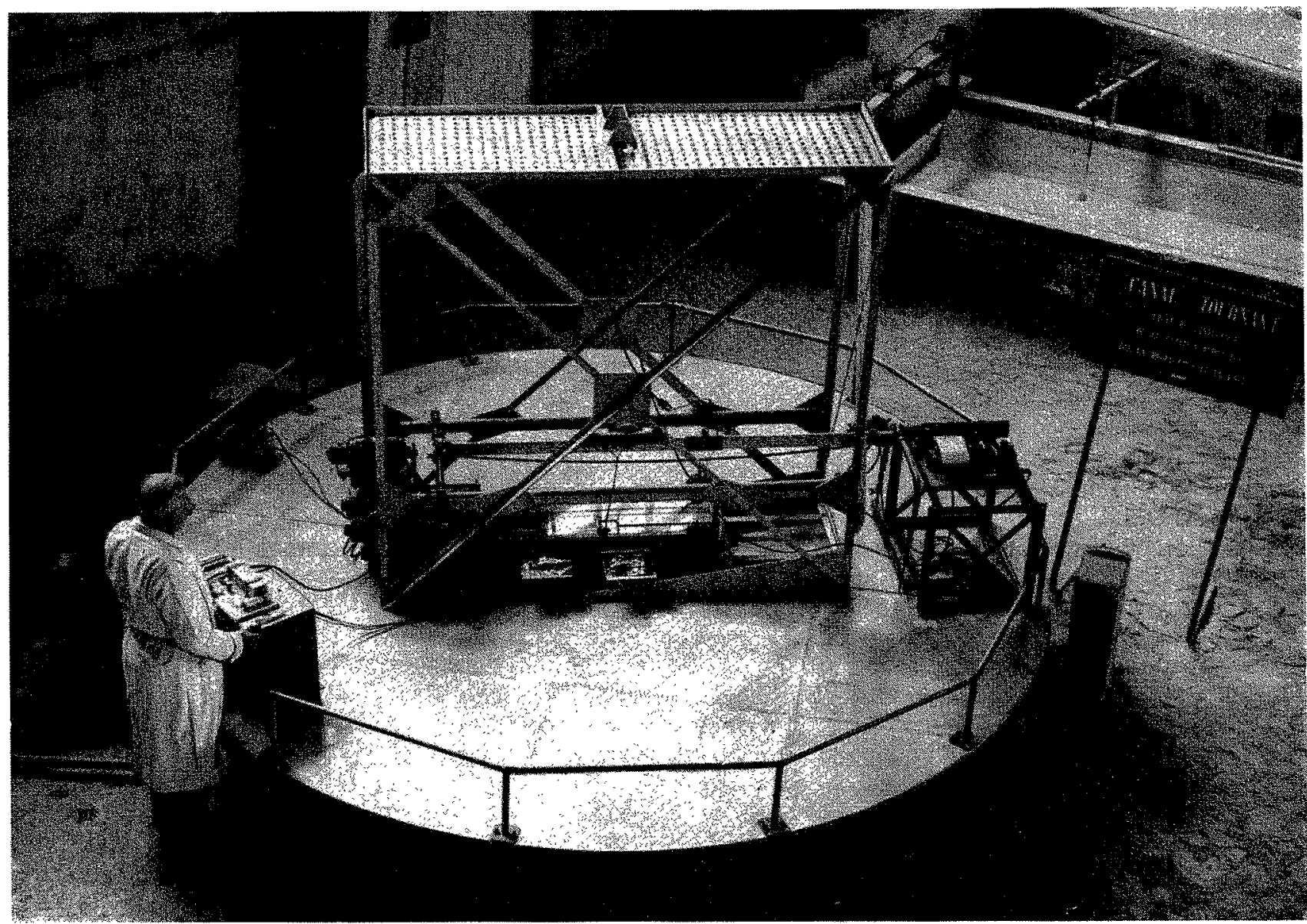

Vue générale du canal tournant. au Laboratoire National d'Hydraulique de Chatou.

ment l'amplitude de l'onde en fonction de l'espace.

- Pour les mesures de vitesse : une caméra électrique de $16 \mathrm{~mm}$ cinématographie le mou- vement de petits flotteurs en polyéthylène placés à la surface de l'eau du bassin. L'étude et la restitution des trajectoires sont obtenues sur l'écran d'un analyseur de film.

\section{III. - CONDITIONS HYDRAULIQUES DES ESSAIS}

La schématisation de la Manche est réalisée dans le canal, en divisant par une paroi la surface du bassin en deux rectangles contigus (fig, 2).

En effet, la longueur totale de la Manche correspond à environ $1 / 2$ longueur d'onde $\lambda$ de la marée semi-diurne calculée pour une profondeur moyenne de $55 \mathrm{~m}(\lambda=1040 \mathrm{~km})$. Dans les mêmes conditions, la largeur de la Manche occidentale est égale à environ $1 / 7$ de la longueur d'onde.

En choisissant les chelles de similitude suirantes :

$$
\begin{array}{r}
\text { - en plan : } 1 / 500000, \\
\text { - en temps : } 1 / 15000, \\
\text { - en hauteur : } 1 / 1100 \text {, }
\end{array}
$$

la profondeur sur le modele doit elre de $50 \mathrm{~mm}$, et la période de marce de 3 secondes. La lon- gueur d'onde de la marée modèle est alors de $2100 \mathrm{~mm}$. La longueur et la largeur de la Manche occidentale doivent être respectivement égales a $525 \mathrm{~mm}$ et $300 \mathrm{~mm}$. La Manche orientale peut être schématiséc par un reclangle de $525 \mathrm{~mm}$ sur $150 \mathrm{~mm}$.

Compte tenu de la situation gégraphique de

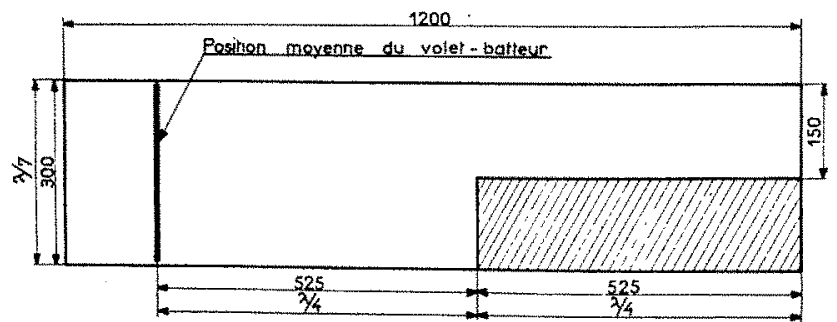

F16. 2. - Plan du bassin sehematisant la Manche (cotes en $\mathrm{mm}$ ) 
la Manche $\left(49^{\circ}\right.$ de latitude nord), la rotation de la plate-forme homologue de la rotation terrestre doit être égale à 1 tour en 8 secondes.

Les marées à l'entrée de la Manche ont une amplitude de $7 \mathrm{~m}$ en vive-eau; en les représentant par une marée de $3,5 \mathrm{~mm}$, on utilise une échelle égale à $1 / 2000$ pour les dénivellations du plan d'eau.

\section{IV. - RÉSULTATS D'ESSAIS}

Nous n'exposerons ici qu'une partie des résullats obtenus sur le canal tournant. en nous bornant à ceux relatifs à la configuration géométrique indiquée précédemment.

Les essais comportent l'étude des marnages et des courants obtenus sur le modèle réduit, en fonction de la vitesse de rotation de la plateforme. Ils ont été réalisés, soit avec un canal à fond lisse, soit avec un canal à fond rugueux.

\section{$1^{\circ}$ ETUDE DE L'ONDE-MARÉE DANS LE CANAL A FOND LISSE}

\section{a) Marnages :}

Les ligures 3 à 5 représentent les cartes des lignes d'égale amplitude de la marée dans les

Canol de $120 \mathrm{~cm}$

Pénode de 10 maree 35

Profondeut $50 \mathrm{~mm}$

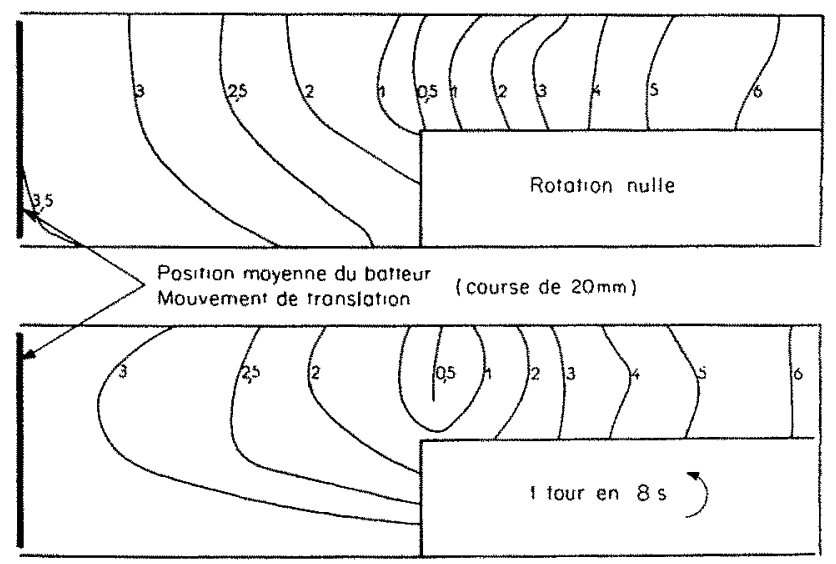

Fir. 3. - Lignes d'égal marnage en $\mathrm{mm}$.

cas : rotation nulle, 1 tour en $8,6,5$ dans un sens ou dans l'autre. Lorsque la plate-forme ne tourne pas, les lignes d'égal marnage dessinent dans la partie schématisant la Manche orientale la forme d'un quart d'onde classique. Dans la partie figurant la Manche occidentale, on obtient un maximum de marnage près du batteur, pujs l'onde diminue graduellement vers la ligne nodale située au droit de Cherhourg.

Lorsque la plate-forme est animé d'un mouvement de rotation, a ligne nodale n'aborde plus le côté du canal schématisant la côte française. Les lignes d'égale amplitude de la marce pren-
Conal de $120 \mathrm{~cm}$ Période de lo morée $3 \mathrm{~s}$ Profondeur $50 \mathrm{~mm}$

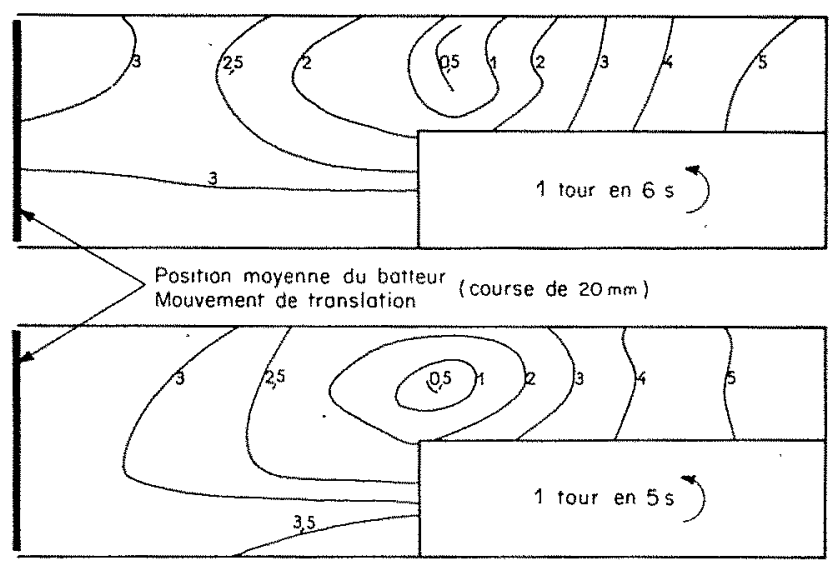

Fic. 4. - Lignes d'egal mamage en $\mathrm{mm}$.

nent une allure elliplique ceinturant une rogion à minimum d'amplitude de l'onde.

Lorsque la rotation du canal a lieu en sens inverse, le point amphidromique est plus netlement visible, il est situé plus près de la côte française.

L'éssentiel des résultats de cette premicre élude des marnages réside dans la transformation de la ligne nodale du $1 / 4$ d'onde, en un point amphidromique d'autant mieux caraclérisé que l'intensilé du vecteur rotalion croit.

On notera que la vitesse de rotation 1 tomr en

Conol de $120 \mathrm{~cm}$

Penrode de la marée 3

Profondeur $50 \mathrm{~mm}$

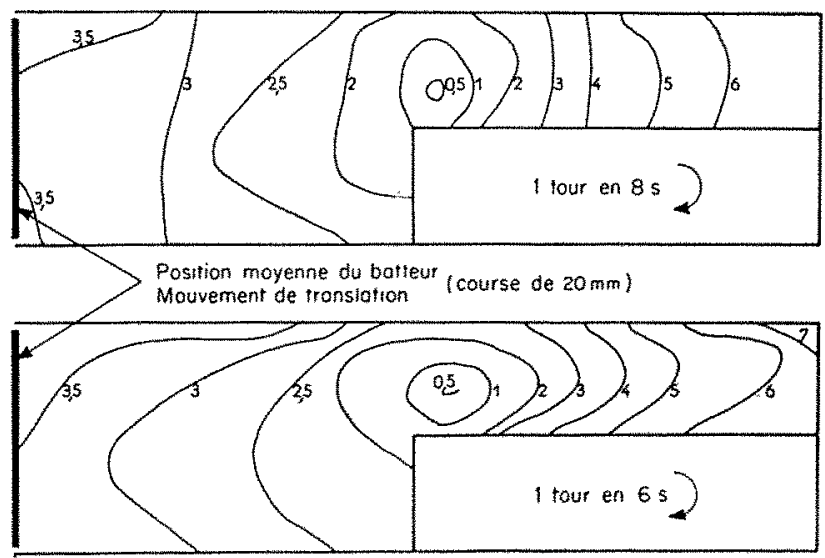

Fu, , - Lignes d'égal marnage en $\mathrm{mm}$. 
6 secondes correspond au cas de la latitude critique. En effet, appelons $\zeta$ la dénivellation due à la marée, $g$ l'accélération de la pesanteur, $h$ la profondeur, $\omega$ l'intensité de la rotation du canal et $\sigma$ la pulsation du mouvement sinusoïdal de la marée. L'équation aux dérivées partielles qui régit le phénomène est la suivante, appelée équation de la membrane :

$$
\Delta \zeta+\frac{\sigma^{2}-4 \omega^{2}}{g h} \zeta=0
$$

Dans le cas particulier d'une rotation égale à un tour en 6 secondes, le terme $2 \omega$ qui traduit l'influence de la force de Coriolis est égal à la pulsation $\sigma$ correspondant à la période de 3 secondes. L'équation ci-dessus n'est plus valable; on dit que l'on se trouve dans les conditions des latitudes critiques.

Les essais ne laissent cependant apparaitre au premier ordre aucun caractère de discontinuité dans la solution physique.

\section{b) Courants :}

La figure 6 reproduit les trajectoires des flotteurs au cours d'une période de la marée. On observe :

- Lorsque la rotation est nulle, les trajectoires ont sensiblement la forme d'ellipses dont le grand axe est parallèle aux côtés longitudinaux du canal;

- Lorsque la plate-forme tourne, il apparaît une circulation des vitesses dans le premier rectangle. Les flotteurs ne décrivent plus des trajectoires fermées, mais tournent indéfiniment autour du centre du rectangle. Cette circulation, liée au rotationnel de la vitesse, est du même sens que la rotation du canal.

\section{$2^{\circ}$ ETUde DE L'ONDE-MARÉ}

OBTENUE DANS LE CANAL AVEG FOND RUGUEUX

La rugosité de fond est réalisée par un quadrillage constitué de bandes de métal déployé de $2 \mathrm{~cm}$ de largeur placées verticalement. Cette disposition est inspirée de la rugosité mise en place sur le fond du modèle de la Manche au 1/50 000 et dont la distorsion est égale à 100 .

\section{a) Marnages :}

Les figures 7 à 10 représentent la carte des lignes d'égal marnage.

- Lorsque la rotation est nulle, la ligne nodale est moins apparente, le minimum d'amplitude est compris entre 1 et $1,5 \mathrm{~mm}$. Le maximum de marnage est situé au fond de l'angle droit schématisant le golfe de Saint-Malo.

- Lorsque la rotation est égale à 1 tour en $8 \mathrm{~s}$,
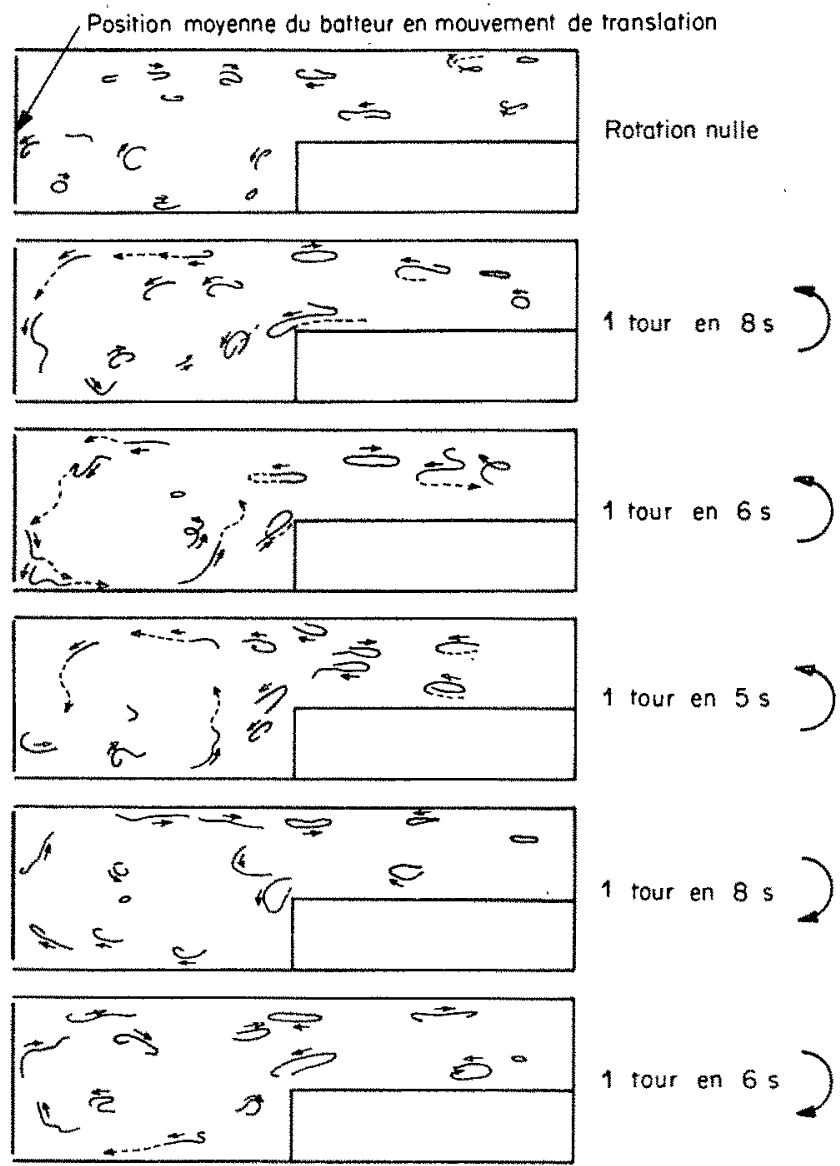

Fig. 6. - Trajectoire d'un flotteur au cours d'une période (trait plein) et d'une partic de la période suivante (trait pointille),

dans le sens inverse des aiguilles d'une montre, c'est-à-dire dans le sens correspondant à l'hémisphère nord, on observe l'apparition d'un point amphidromique virtuel. Dans la partie occidentale de la Manche, les lignes d'égal marnage sont sensiblement parallèles

Conal de $120 \mathrm{~cm}$

Periode de marée 3

Profondeur $50 \mathrm{~mm} \quad$ Essol avec rugosite de fond

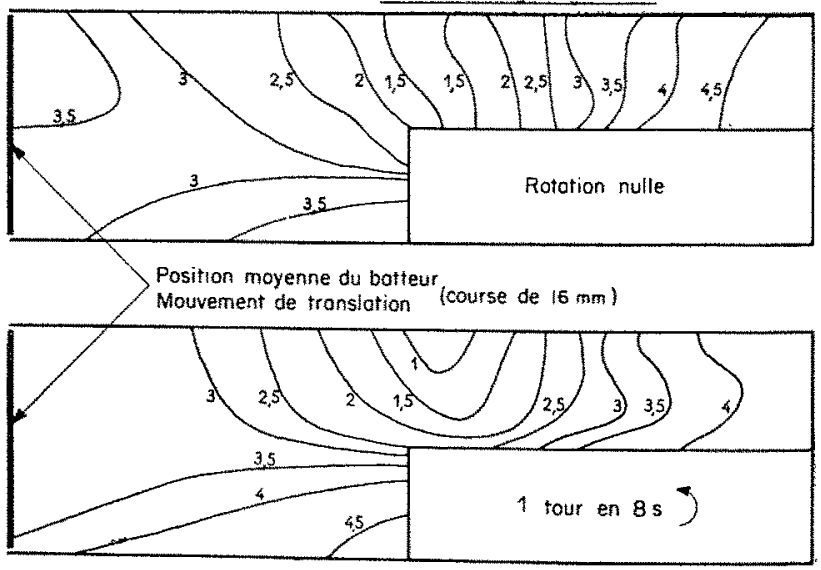

Fis. 7. - Lignes d'ègal marnage én $\mathrm{mm}$. 
à la còte, on retrouve un maximum d'amplitude au fond du golfe de Saint-Malo. La comparaison de la deuxième carte de la figure 6 à la carte de la figure 12 , représentant les lignes d'égal marnage de la marée dans la nature, montre que la représentation de la marée sur le modèle réduit schématique peut être considérée comme très satisfaisante. Cette dernière comparaison montre l'influence

Conal de $120 \mathrm{~cm}$

Période de la morée $3 \mathrm{~s}$

Profondeur $50 \mathrm{~mm}$

Esso: ovec rugosité de fond

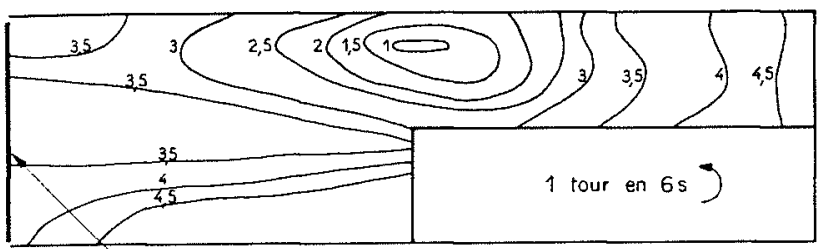

Position moyenne du botteur

Mouvement de tronstotion (course de $16 \mathrm{~mm}$ )

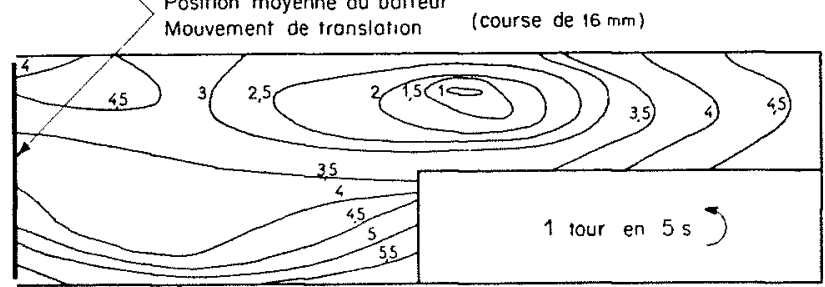

Fig. 8. - Lignes d'égal marnage en $\mathrm{mm}$.

prépondérante du frottement sur la propagation de la marée dans la Manche. Ce résultat avait déjà été prévu par l'étude des considérations énergétiques de la marée en Manche [3].

- Lorsque la vitesse de rotation augmente, le point amphidromique bien caractérisé devient réel (fig. 8).

- Enfin, dans le cas où la rotation s'effectue en sens inverse (fig. 9), on observe la formation d'un point amphidromique près du sommet

Canal de $120 \mathrm{~cm}$

Période de la marée 3

Profondeur $50 \mathrm{~mm} \quad$ Essai ovec rugosité de fond
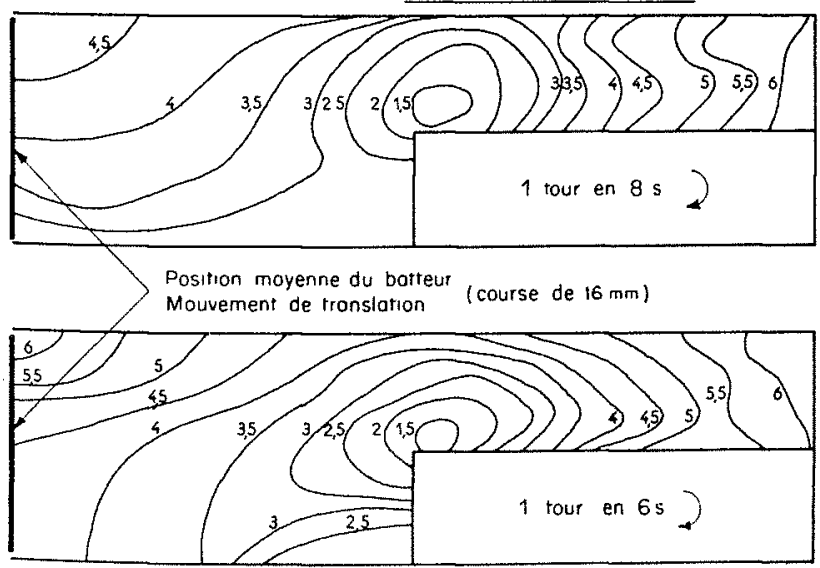

Fic. 9. - Lignes d'égal marnage en $\mathrm{mm}$, du rectangle au large de Cherbourg. Le maximum de marnage en Manche occidentale est situé du côté anglais. Il n'y a plus de maximum de marnage au fond du golfe de SaintMalo.

Compte tenu de la représentation assez fidèle de la nature dans le cas de l'hémisphère nord,

Conal de $120 \mathrm{~cm}$

Profondeur $50 \mathrm{~mm} \quad$ Essai ovec rugosité de fond

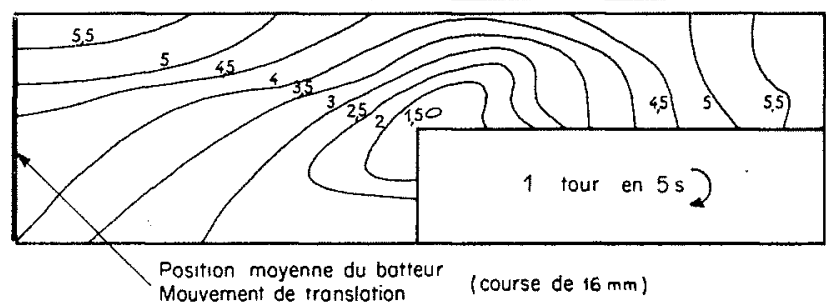

Fig. 10. - Lignes d'égal marnage en mm.

on peut dire que la première carte de la figure 9 ( 1 tour en $8 \mathrm{~s}$ ) représente les lignes d'égal marnage que l'on obtiendrait si la Manche était située dans l'hémisphère sud.

\section{b) Courants :}

Sur la figure 11 sont tracées les trajectoires des flotteurs. On remarque principalement que les trajectoires ont toujours sensiblement la

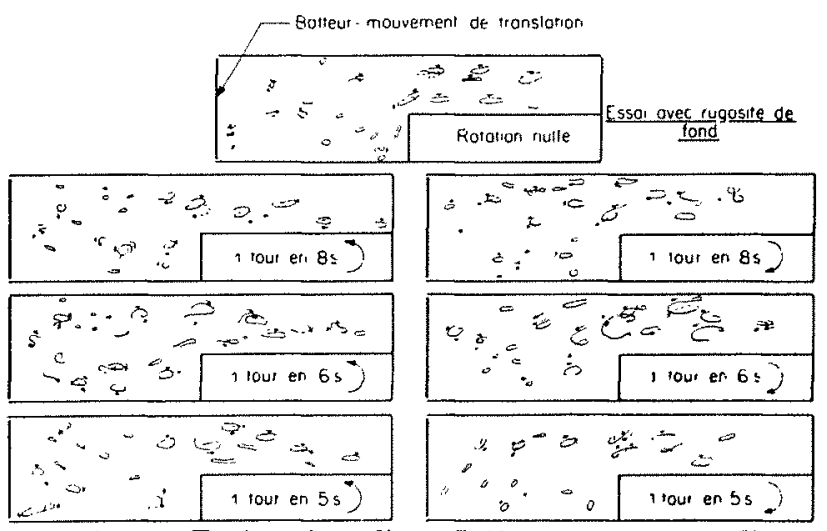

Fig. 11. - Trajectoire d'un flotteur au cours d'une période (trait plein) et d'une partie de la période suivante (trait pointillé).

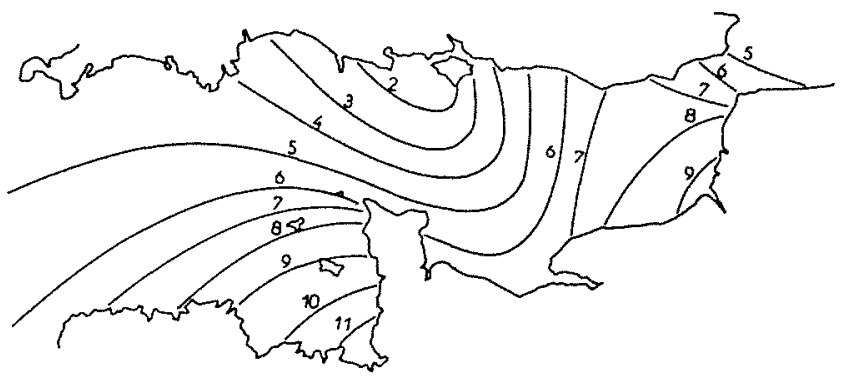

Fia. 12. - Ligne d'égal marnage en mètres dans la Manche (marée de vive-eau moyenne). 
forme d'ellipse aplatie. Lorsque la plate-forme tourne, il n'apparaît pas de circulation. Enfin, dans le cas ou la rotation a lieu dans le sens inverse des aiguilles d'une montre, les courants sonl plus importants du côté de la côte francaise que du côté de la côte anglaise. Ce résultat est conforme aux données naturelles et à la theorie des ondes-marées de Kelvin [4] dans un canal. La circulation des courants est nulle, leur intensité est plus forte du côté où le marnage est maximum. L'examen des figures fait apparâttre aussi un minimum de courant (ellipse très petite) près de la côte anglaise au milieu du premier rectangle, là où la théorie préroit l'existence d'un point mort pour le courant. Ces résultats sont inversés lorsque la rotation a lieu en sens contraire, le point mort de courant est situé près de la cote française.

\section{V. - CONCLUSION}

Il ressort de l'énoncé de ces quelques résultats obtenus lors de l'étude de la marée sur un canal tournant, et relatifs au cas particulier de de la Manche, que :

$1^{*}$ La force de Coriolis ne permet pas à elle seule d'expliquer suffisamment le phénomène de la marée en Manche, puisque d'une part elle n'est pas la cause de l'augmentation de l'amplitude de la maréc dans le golfe de Saint-Malo, et que d'autre part elle produirait un point amphidromique réel.

$2^{\circ}$ L'introduction de la force de frottement est suffisante pour obtenir l'augmentation du marnage dans le golfe de Saint-Malo, mais elle n'introduit pas la disparité des marées entre les côtes françaises et anglaises.

$3^{*}$ La présence simultanée des forces de Corio- lis et de frottement a pour eflet : de cré un point amphidromique virtuel et par voie de conséquence d'engendrer la disparité des marées françaises et anglaises, d'oì une représentation assez correcle du phénomène naturel sur le modèle réduit schématique.

$4^{\circ}$ Le fait que la forme de l'onde-marée dans le golfe de Saint-Malo soit surtout influencée par la rugosité plus que par la forcè de Coriolis, permet de donner plus de poids aux résultats obtenus sur un modèle réduit fixe en ce qui concené la région du golfe de Saint-Malo. Or, c'est cette région, et elle seule, qui est intéressante à connaitre et à reproduire sur modèle réduit pour les études de l'aménagement marémoteur des îles Chausey.

\section{BIBLIOGRAPHIE}

1. X. Mrohon et R. Bonneflile. - k La marée dans la Manche, Construction et réglage d'un modèle réduit.» (Quatrièmes Journées de l'Hydrauliq̨ue, question IV, rapport $n^{\circ} 4$, Paris, 1956 .)

2. L. VANTrovs. - "le remous d'un ouvrage dans une mer à marée. (Thèse de Doctorat ès seiences, Paris, 1957,
3. R. Bonnefiles. - - Etude de 1a mare en Manehe. (Quatrièmes Journées de l'Hydraulique, question II, rapport $n^{\circ} 5$, Paris, 1956 .)

4. E. Fichot. - « Expose critique de la théorie des matrées. (Annales du Burcau des Longitudes, tome $\mathrm{XI}, 1938$.

DI S C S S I O N

président : M. Gubrat

M. lo President remereie M. Bonnefules et souligne lintérêt de ses travaux, limportance el la nouveauté des resultats qu'il vient d'exposer, th saroir que, sous réserve de vërifications plus poussées, les fortes marées du golfe de Saint-Malo seraient en correspondance avec les phénomènes de frottement. L'intégration de ces résultats permettra de mieux comprendre le régime des marées et, par conséguent, de connaître avec plus de certitude les déformations que l'usine marẻmotrice prévue dans la baie de Saint-Malo est susceptible d'apporter.
A ce propos, M. le Président signale que, pout la première fois dans l'histoire du monde, des groupes marem moteurs, eeux qui ont été installés à titre dessai a Cambeyrac et à Argentat, ont été mis recemment en route dans ces usines.

M. Ginent demande pourquoi on a tant schématisé la representation de la Manche, alors qu'il etait aussi facile sur modele de mieux se rapprocher de la confguration naturelle.

M. Bonnefilte répond que la représentation schèmati- 
que de la Manche a été adoptée, en raison du peu de confiance que l'on avait, a priori, dans le modèle et que l'on espérait reprendre l'étude par le calcul.

M. le Président ajoute que M. Bonnefille a fait ec calcul suivant l'idée de M. Lacome et dans des eireonstances très voisines de la réalité.

M. Gribnt se demande, à propos de l'influence prépondérante du frottement pour I'explication de la marée dans la baie de Saint-Malo, s'il en sera de même quand on étudiera l'influence du projet du Mont Saint-Michel sur la maréc au roisinage de la côte anglaise où existe un point amphidromique virtuel. Il pense qu'à ce moment on ne pourra plus négliger la force de Coriolis.

M. le Président indique qu'un programme de recherches, à la fois par le calcul et sur modèles, est actuellement adopté en vue de préciser, entre autres choses, si la force de Coriolis peut être négligée dans l'étude de l'influence du projet du Mont Saint-Michel au voisinage de la côte anglaise. M. Bonnefille pense que cette force peut être négligée dans le cas de l'usine de Chausey.

M. Biesex demande sur quelles bases il est possible de comparer "l'amplification» de modèles différents (tournant ou non, rugueux ou non, à profondeur constante ou non, etc...). Cette 《amplification》 est le rapport entre l'amplitude dans le golfe de Saint-Malo et une certaine amplitude de référence. Que faut-il choisir comme amplitude de référence? Ce choix ne peut être arbitraire sans infirmer la validité des comparaisons. Par exemple, dans la nature, selon que l'on prendra l'amplitude de référence sur les côtes anglaises ou sur les côtes francaises (disons, dans les deux cas, vers l'entréc de la Manche) «l'amplification 》 variera du simple au double. Dans un modèle non tournant (ou non rugueux) ce même choix n'introduira qu'une différence minime dans les amplifications calculées. La comparaison des coefficients d'amplification de modèles fixes d'une part, et tournants d'autre part, risque done de donner des résultats très différents suivant le ehoix du point de référence.

M. Bonnefilte indique que, dans le golfe de Saint-Malo et dans Ie modèle tournant où la profondeur est constante, il n'a été constaté une augmentation du marnage que lorsqu'il y a frottement; dans le modèle fixe, où i y a une diminution des profondeurs, et de la rugosité de fond, on obtient une augmentation de mainage qui est du même ordre que celle qui a été obtenue dans le modèle tournant et dans des conditions semblables par ailleurs.

M. Vanthoys indique que l'exposé de M. Bonnefille apporte, entre autres choses, un élément de réponse à la question précédemment soulevée, ce matin même par M. Chapoutier. (1)

Quelle est en effet la signification «physique» du cas de la rotation «critique» mis en évidence dans la discussion de l'écuation des marées?

M. Bonerider nous a montré des schémás représentant le réseau des courbes d'égalc amplitude d'une maréc sinusoüdale dans les trois cas oủ la rotation est hypocritique, critique ou hypercritique : aucune discontinuité ni aucune singularité caractéristique du point de vue physique n'apparaissent dans le passage d'un cas à l'autre.

Il semble qu'on puisse en conclure que la singularité analytique attachée à une certaine valeur critique de la rotation n'a pas d'incidence physique tangible. Cette conclusion subsisterait vraisemblablement, même si l'on lient compte du fait qu'il faudrait considérer non pas le schéma expérimental lni-même (la rugosité ne peut pas y être rigoureusement nulle), mais la limite vers laquelle tend co schéma quand la rugosité tend vers zéro.

Par contre, ect aspect singulier du cas critique se relrouve, sinon dans le phénomène lui-même, du moins

(1) Voir discussion de la communication de M. VANTnoxs. dans le mode de représentation sur maquette de laboratoire que M. Vantroxs en a proposé (distorsion des profondeurs selon une formule qui, en l'absence de rasosité, conduirait à une profondeur-image infiniment grande dans le cas critiquc, ou à une profondeur-image négative dans te cas hypercritique si le modèle ne tourne pas).

M. le Président précise que, pour interpréter physiquement le cas «critique», il manque une analogie avec l'expérience propre. Ce n'est donc, peut-titre pas à proprement parler un cas critique, puisque l'on ne trouve un sens physique à cette critiqualité que dans la profondeur infinie qui se manifeste, non dans la nature, mais seulement dans l'étude sur modèle avec déformation : on ne devine pas très bien le sens qu'il peut $y$ avoir, dans une relation simple entre la vitesse de rotation et la force de Coriolis.

M. Surrel propose, pour aider à comprendre cette critiqualité, la comparaison avec le phénomène qui se manifeste quand on étudie un système oscillant dans un circuit électrique : en négligeant les résistances, on obtient des surtensions et en les prenant en compte, on a des chanees de retrouver les choses.

M. Lhenmtre rappelle que, si mathématiquement, il y a indetermination en faisant intervenir la rugosité, on obtient une solution pratique lors du passage à la limite.

M. Bonnefille se demande s'il n'y a pas une différence du second ordre que des mesures plus poussées pourraient révéler : lorsqu'on tourne vite, les ondes sont plus stables par suite de la disparition des parasites; or dans un cas hypereritique, il n'y a pas tharmoniques et seule subsiste la fonction propre de la marce.

M. Baxar, attire l'attention sur l'intérêt de certains essais, qui ont été effectués avec une cuve rectangulaire dont M. BONNEFILLE n'a pas parlé.

Sans rugosité ni rotation, on a obtenu un mouvement stationnaire cylindrique arec une ligne nodale au milied (un résultat très analogue aurait été certainement obtenu avec rugosité sans rotation).

Sans rugosité anec rotation, on a obtenu un mouvement stationnaire avec un point nodal au centre du rectangle sans différence de marnage sur les frontières nord et sud. Il parait évident en effet, que la force de Coriolis ne peut faire apparaître de dissymétrie dans le sens nordsud, alors que le mouvement est symétrique dans le sens est-ouest.

Il paraît possible de prévoir quel aurait élé le résulfat d'un essai (non réalisé) effectué avec rotation et rugosité.

La rugosité introduisant une dissymétric longitudinale, ic mouvement ne serait plus purement stationnaire, mais partiellement progressif et l'effet de la force de Coriolis sur la part progressive ferait apparaitre une différence d'amplitude entre le nord et le sud analogue a celle constatée dans l'onde de Kelvin.

La différence des marnages existant sur la côte anglaise ef la côte française rẻsulte done bien de la force de Coriolis, mais ne se produit que si la rugosité existe.

M. Bonnefirte rappelle que dans le cas ou l'essai ne
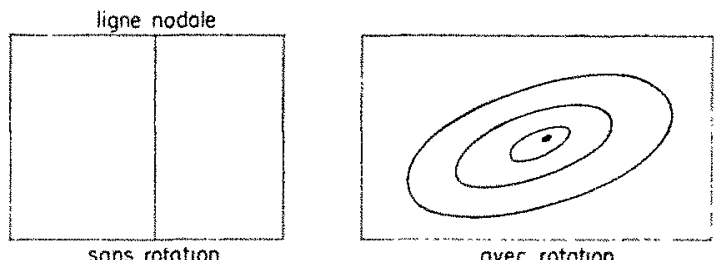

porte que sur la côte française, avec Cotentin schématisé, on obtient le maximum de marnage vers Saint-Malo : si Fon réduit la Manche à un eanal rectangulaire, on obtient une ligne nodale dans le cas sans frottement et sans rotation, et un point amphidromique dans le eas alece rotation. 